\title{
Análisis de la evolución de las Asesorías Académicas en el periodo 2014-2019 en una DES
}

Analysis of the evolution of the Academic Advisors in the 2014-2019 period in a DES

SORDIA-SALINAS, Cesar†, PALOMARES-RUIZ, María Blanca, TORRES-BUGDUD, Arturo y TREVIÑO-CUBERO, Arnulfo

Universidad Autónoma de Nuevo León, Facultad de Ingeniería Mecánica y Eléctrica

ID $1^{\text {er }}$ Autor: Cesar, Sordia-Salinas / ORC ID: 0000-0003-2186-1080, Researcher ID Thomson: S-5666-2018, CVU CONACYT ID: 339888

ID $1^{\text {er }}$ Coautor: María Blanca, Palomares-Ruiz / ORC ID: 0000-0002-4079-6969, Researcher ID Thomson: S-4843-2018, CVU CONACYT ID: 339594, arXiv ID: Mabe_6708

ID $2^{\text {do }}$ Coautor: Arturo, Torres-Bugdud / ORC ID: 0000-0003-2214-9394, CVU CONACYT ID: 216332
ID $3^{\text {er }}$ Coautor: Arnulfo, Treviño-Cubero / ORC ID: 0000-0002-0958-8352, CVU CONACYT ID: 373815

DOI: $10.35429 / J E T .2020 .11 .4 .7 .12$

Recibido: 28 de Marzo, 2020; Aceptado 29 de Junio, 2020

\begin{abstract}
Resumen
Preocupados por una educación de calidad, con retos de una creciente incertidumbre sobre el futuro de las Instituciones de Educación Superior, vencer el desafío de ingresar y transitar exitosamente en este nivel de estudios a los estudiantes, ha sido motivo del presente análisis, en las instituciones académicas es un efecto que se ha estado generalizando y extendiendo, lograr un resultado donde se consiga hacer perdurar lo aprendido, comprendiendo los temas que se abordan en clase, la Asesoría Académica ha sido, en efecto, el recurso para orientar al alumno. El propósito es obtener el éxito escolar con el Programa Institucional de Asesorías Académicas en una Dependencia de Educación Superior, por lo que el análisis de la tendencia y evolución de la aprobación y reprobación la forma en la que se ha llevado a cabo mediante la recolección de datos acerca de los índices de reprobación, aprobación, población de asesorados en los cursos impartidos. Una vez obtenidos dichos datos se procedieron con su organización y análisis mediante un modelo estadístico que nos demuestra el impacto positivo a su vez se cumple un periodo de contribuir a mejorar los indicadores de la competitividad académica de la Dependencia.
\end{abstract}

Asesorías Académicas, Deserción, Reprobación

\begin{abstract}
Concerned about a quality education, with challenges of growing uncertainty about the future of Higher Education Institutions, overcoming the challenge of entering and successfully transitioning students to this level of studies, has been the reason for this analysis, in the institutions Academic studies is an effect that has been generalizing and spreading, achieving a result where what has been learned can be made to last, understanding the issues that are addressed in class, Academic Advising has been, in effect, the resource to guide the student. The purpose is to obtain school success with the Institutional Program of Academic Counseling in a Higher Education Unit, so that the analysis of the trend and evolution of approval and disapproval is the way in which it has been carried out by collecting data about the failure rates, approval, population of advised in the courses taught. Once these data were obtained, they proceeded with their organization and analysis using a statistical model that shows us the positive impact, in turn, there is a period of contributing to improve the indicators of the academic competitiveness of the Unit
\end{abstract}

Academic Advising, Dropout, Failure

Citación: SORDIA-SALINAS, Cesar, PALOMARES-RUIZ, María Blanca, TORRES-BUGDUD, Arturo y TREVIÑOCUBERO, Arnulfo. Análisis de la evolución de las Asesorías Académicas en el periodo 2014-2019 en una DES. Revista Teoría Educativa. 2020. 4-11: 7-12

\footnotetext{
$\dagger$ Investigador contribuyendo como primer autor.
} 


\section{Introducción}

La iniciativa Futuros de la educación de la UNESCO tiene como objetivo repensar la educación y dar forma al futuro. La iniciativa está catalizando un debate global sobre cómo el conocimiento, la educación y el aprendizaje deben reinventarse en un mundo de creciente complejidad, incertidumbre y precariedad, preocupados y ocupados por la promoción de una enseñanza y un aprendizaje de calidad. (UNESCO, 2020)

En este contexto, diversos estudios de investigadores educativos preocupados por los indicadores de la calidad educativa en sus instituciones, se han dado a la tarea de emplear múltiples estrategias para combatir la deserciónreprobación escolar, aunado a estudiar tendencias de eficiencia terminal que se reflejan en altos costos para las mismas, todas con el propósito de disminuir el abandono y elevar las cifras a niveles satisfactorios no solo de una institución, sino que contribuye para todo el país.

Como lo describe Carreón et al (2014) en su artículo Disminución del índice de reprobación mediante estrategias tutoriales, realiza un análisis sobre diversos estudios que comprenden la relación deserción-reprobación escolar señalando una relación significativa entre ambas, además citando entre otros a Ocampo et al.(2010), la deserción y la reprobación de los estudiantes universitarios de las carreras de ingeniería han pasado a ser, una gran preocupación en las instituciones de educación superior en México, haciendo referencia que la deserción-reprobación alcanzó hasta $60 \%$, y que la deserción en las universidades públicas se calcula en $58 \%$, mientras que en las privadas en $39 \%$. Además, menciona que el abandono de los estudios universitarios ha tenido un costo de entre $141 \mathrm{y}$ 415 millones de dólares.

Las investigaciones en relación a la Eficiencia Terminal de acuerdo a Toscano de la Torre (2015) han identificado una diversidad de factores que influyen en el logro de la Eficiencia Terminal de un estudiante en la Educación Superior; dentro de este conjunto de factores, los más recurrentes son:

El modelo educativo, el perfil del estudiante, la reprobación, la responsabilidad, programas de estudios obsoletos, el lugar de residencia, falta del dominio del inglés, factores económicos, factores psicológicos y fisiológicos, la familia, la trayectoria académica, la vinculación institucional citando a (Casillas, Alvarado y Cols., 2011), (García Robelo y Barrón Tirado, 2009), (Lara Barrón y Valadez Díaz, 2011), (Sánchez, Figueroa y Cols. 2013), (Navarro, 2004), (Toscano, de la Torre y Cols. 2015)

Como lo indica la SEP (2019), en lo que se refiere a la eficiencia terminal 2018-2019 sus indicadores a nivel medio superior son:

2018-2019

\begin{tabular}{|c|c|c|c|}
\hline Entidad & Total & Mujeres & Hombres \\
\hline Republica Mexicana & 63.9 & 68.4 & 59.5 \\
\hline 2018-2019 & & \multicolumn{2}{|c|}{ Abandono Escolar (\%) } \\
\hline Entidad & Total & Mujeres & Hombres \\
\hline Republica Mexicana & 14.5 & 12.6 & 16.4 \\
\hline
\end{tabular}

Con base en lo anterior, se puede observar que se han realizado enormes esfuerzos por diseñar estrategias para incrementar la calidad educativa, con acciones para combatir la deserción escolar y la reprobación, buscando obtener mejores índices de aprovechamiento y de eficiencia terminal encontrando a las asesorías académicas como una de las acciones para afrontar los altos índices de reprobación.

Entre otras propuestas Fernández (2017) señala que para que se cumplan con los estándares de calidad que ya mantienen las instituciones en sus programas existentes, y que este crecimiento se lleve a cabo en programas académicos relevantes para el desarrollo local y regional, recomienda fortalecer la infraestructura que favorece el logro educativo de los estudiantes, así como promover programas de acompañamiento y de incentivos para que los estudiantes concluyan satisfactoriamente sus estudios.

Como señala (Verónica Corona, 2016) y sus co-autores en su artículo "Estrategias para la disminución de los índices de reprobación en el Instituto Tecnológico de Pachuca.": 
"Las asesorías deben ser parte importante de las estrategias para afrontar el alto índice de reprobación. Éstas deben impartirse por cada profesor que esté frente a una materia, debe estar dispuesto a apoyar a los alumnos en una situación problemática, así como establecer un horario y un lugar específico; dentro de la asesoría el docente estará dispuesto a resolver dudas académicas."

La autora recomienda los siguientes puntos para llevar una asesoría exitosa:

- Las asesorías deben realizarse en un lugar espacioso, ventilado y con suficiente iluminación.

- $\quad$ Es importante programar las asesorías y la planeación de las mismas, contemplando el horario de sus alumnos.

- El asesor deberá conducirse con claridad, sencillez y veracidad en la información que dé a conocer, así como tomará interés de lo que le planteen sus estudiantes.

Por su parte en la Facultad de Ingeniería Mecánica y Eléctrica (FIME) de la Universidad Autónoma de Nuevo León (UANL), ha estado llevando a cabo el programa de asesorías académicas cuyo propósito es contribuir a disminuir el alto índice de reprobación contando con la colaboración de profesores dispuestos a apoyar a los estudiantes en las unidades de aprendizaje que les han causado dificultad aprobar, cuyo factor primordial ha sido la disponibilidad de horario que cada docente ha registrado ante la Coordinación de Asesorías.

En estudios anteriores nos hemos planteado diversas preguntas como ¿Qué tipo de impacto han tenido las asesorías académicas en la FIME?, ¿Se ha cumplido el objetivo de reducir el índice reprobatorio? ¿Existe una relación entre brindar asesoramiento y la reducción del índice de reprobación dentro de la Dependencia? El propósito fundamental es obtener el éxito escolar en los estudiantes que han utilizado el programa de Asesorías Académicas de la FIME, aunado al análisis de la tendencia y evolución de la aprobación y reprobación a la forma en la que se han llevado a cabo, reuniendo información del periodo comprendido desde 2014 a 2019 este artículo ha sido elaborado con la información de la base de datos proporcionada por la FIME, en su búsqueda por mejorar los indicadores en la competitividad académica de la Dependencia.

\section{Metodología}

El estudio de la evolución y tendencia de las asesorías académicas se realiza durante cada semestre en el periodo que comprende AgostoDiciembre 2014 a Enero Junio 2019, como se presentó en Sordia,et al (2018) se utiliza el método cuantitativo, el cual según (Roberto Hernández Sampieri, 2004) es: "la que nos ofrece la posibilidad de generalizar los resultados más ampliamente, nos otorga control sobre los fenómenos y un punto de vista de conteo y magnitudes de éstos.

Asimismo, nos brinda una gran posibilidad de réplica y un enfoque sobre puntos específicos de tales fenómenos, además de que facilita la comparación entre estudios similares." con base a esto, se llevó acabo la recolección de datos acerca de los índices de reprobación, aprobación, asesorados y población en los cursos impartidos dentro de la FIME. Una vez obtenidos dichos datos se procedió con su organización y análisis mediante un modelo estadístico, el cual ayudó a exponer si la hipótesis que menciona una relación entre brindar asesoramiento y la reducción del índice de reprobación dentro de la dependencia, se cumple o no. A continuación, dentro del apartado de resultados se explica más a detalle los pasos y resultados de la investigación.

\section{Resultados}

A continuación, se presenta una estadística de resultados que fue realizada a partir de una base de datos, los cuales son registrados semestralmente por la Coordinación de Asesorías y de la Coordinación de Capacidad Académica de la FIME, donde se puede consultar información relevante como:

\section{- $\quad$ Población de la FIME.}

- Asesorados.

- Programa educativo (carrera).

- $\quad$ Unidad de aprendizaje (materia).

- Área (ciencia básica, especialidad de carrera o formación general universitaria).

- Aprobó / reprobó (situación del estudiante) 
En el gráfico 1 se muestra los periodos de actividad de asesorías de la FIME, en los cuales se observa que periodo tras periodo, existe una relación entre la población de la facultad y la cantidad de alumnos asesorados. Este comportamiento se puede determinar gracias al método de la regresión lineal simple, el cual nos dice como ha sido la tendencia con una línea que se grafica bajo una pendiente con una ecuación que se puede calcular con los datos que aparecen en la Tabla 1.

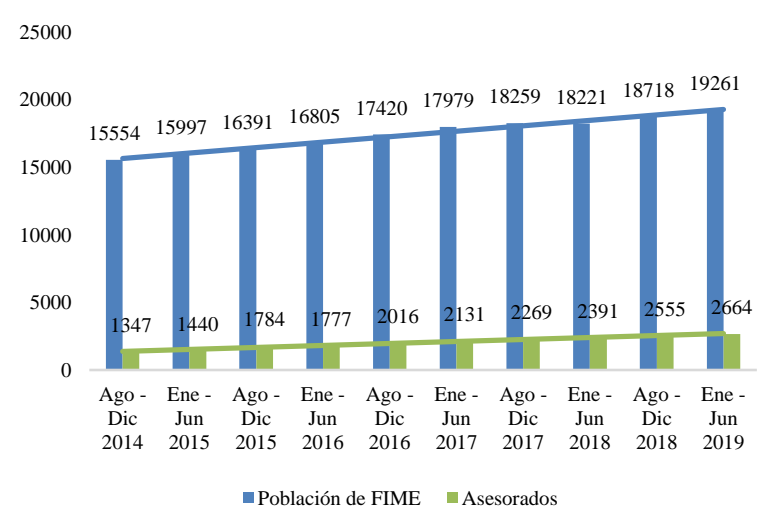

Gráfica 1 Análisis comparativo Población de la FIME y número de estudiantes Asesorados VS el periodo semestral

Fuente: Elaboración Propia

\begin{tabular}{|l|r|r|r|r|}
\hline $\begin{array}{c}\mathbf{x} \\
\text { (periodo) }\end{array}$ & \multicolumn{1}{c}{$\begin{array}{c}\mathbf{y} \\
\text { (alumnos) }\end{array}$} & \multicolumn{1}{c|}{\begin{tabular}{c} 
X.y \\
\multicolumn{1}{|c|}{$\mathbf{X}^{\mathbf{2}}$}
\end{tabular}} & \multicolumn{1}{c|}{$\mathbf{Y}^{\mathbf{2}}$} \\
\hline 1 & 15,554 & 15554 & 1 & 241926916 \\
\hline 2 & 15,997 & 31994 & 4 & 255904009 \\
\hline 3 & 16,391 & 49173 & 9 & 268664881 \\
\hline 4 & 16805 & 67220 & 16 & 282408025 \\
\hline 5 & 17420 & 87100 & 25 & 303456400 \\
\hline 6 & 17979 & 107874 & 36 & 323244441 \\
\hline 7 & 18259 & 127813 & 49 & 333391081 \\
\hline 8 & 18221 & 145768 & 64 & 332004841 \\
\hline 9 & 18718 & 168462 & 81 & 350363524 \\
\hline$\Sigma=$ & 19261 & 192610 & 100 & 370986121 \\
\hline 10 & 174605 & 993568 & 385 & 3062350239 \\
\hline
\end{tabular}

Tabla 1 "Población de FIME"

Fuente: Elaboración Propia

En la Tabla 1 "Población de FIME" donde:

$\mathbf{x}=$ Número del periodo que se cursó.

$\mathbf{y}=$ La cantidad de alumnos en la FIME.

$\mathbf{x}^{*} \mathbf{y}=$ La multiplicación de la columna " $\mathrm{x}$ " por la columna "y" (en la misma fila).

$\mathbf{X}^{\mathbf{2}}=$ El cuadrado del número en la columna " $\mathrm{X}$ ". $\mathbf{Y}^{\mathbf{2}}=$ El cuadrado del número en la columna " $\mathrm{y}$ ".

$\mathbf{N}=$ Número de periodos.

$\Sigma=$ Sumatoria de las columnas.

$\overline{\mathbf{x}}=\Sigma \mathrm{x} / \mathrm{N}$

$\overline{\mathbf{Y}}=\Sigma \mathrm{y} / \mathrm{N}$

$\boldsymbol{\sigma x y}=[\Sigma(\mathrm{x} * \mathrm{y}) / \mathrm{N}]-[\overline{\mathrm{x}} * \overline{\mathrm{Y}}]$

$\sigma \mathbf{x}^{2}=\left[\Sigma\left(\mathrm{x}^{2}\right) / \mathrm{N}\right]-[\overline{\mathrm{x}}]$

$\sigma \mathbf{y}^{2}=\left[\Sigma\left(\mathrm{y}^{2}\right) / \mathrm{N}\right]-[\overline{\mathrm{Y}}]$

ISSN 2523-2509

ECORFAN® Todos los derechos reservados

\section{Ecuación:}

$\mathrm{Y}-\overline{\mathrm{Y}}=\left[\sigma \mathrm{xy} / \sigma \mathrm{x}^{2}\right] *(\mathrm{x}-\overline{\mathrm{x}}) *$ Ecuación $\mathrm{l}^{*}$

Entonces obtendremos que la ecuación será:

$\mathrm{Y}=100.728787 \mathrm{X}+16906.4917$

De la misma manera con la cual se realizó el Gráfico No. 1 para poder obtener los incrementos en la población de la FIME y de sus asesorados, se llevó acabo el Gráfico No. 2, donde se muestra un análisis comparativo entre la cantidad de alumnos asesorados que tuvieron un resultado positivo (Aprobación) o por ende uno Negativo (Reprobación) dicho gráfico se realizó con los datos obtenidos en la Tabla No. 2. "Asesorados" que se muestra a continuación.

\begin{tabular}{|r|r|r|r|r|}
\multicolumn{1}{c}{$\mathbf{x}$ (periodo) } & $\mathbf{y}$ (alumnos) & \multicolumn{1}{c|}{$\mathbf{x y}$} & $\mathbf{X}^{\mathbf{2}}$ & $\mathbf{Y}^{2}$ \\
\hline 1 & 1347 & 1347 & 1 & 1814409 \\
\hline 2 & 1440 & 2880 & 4 & 2073600 \\
\hline 3 & 1784 & 5352 & 9 & 3182656 \\
\hline 4 & 1777 & 7108 & 16 & 3157729 \\
\hline 5 & 2016 & 10080 & 25 & 4064256 \\
\hline 5 & 2131 & 12786 & 36 & 4541161 \\
\hline $5=$ & 2269 & 15883 & 49 & 5148361 \\
\hline 7 & 2391 & 19128 & 64 & 5716881 \\
\hline 8 & 2555 & 22995 & 81 & 6528025 \\
\hline 9 & 2674 & 26740 & 100 & 7150276 \\
\hline 10 & 20384 & 124299 & 385 & 43377354 \\
\hline 55 & & & & \\
\hline
\end{tabular}

Tabla 2 "Asesorados"

Fuente: Elaboración Propia

\section{$Y=61.7696 X+1698.6666$}

Se puede observar que para las dos ecuaciones la ecuación tiene una pendiente positiva por lo tanto la tendencia será aumentar mientras los periodos continúen.

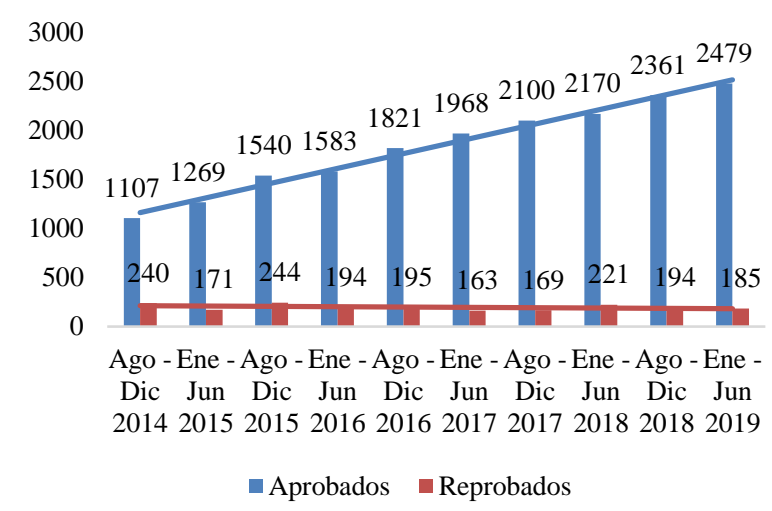

Gráfica 2 Análisis Comparativo de alumnos Asesorados con reprobación vs aprobación Fuente: Elaboración Propia 
Ahora bien, en el gráfico 2 se muestran los estudiantes aprobados y reprobados por cada periodo, y haciendo relación al gráfico 1 se muestra una línea tendencia de los resultados. La parte de los aprobados es representada con una línea azul, en la cual la tendencia es positiva, mientras la parte de los alumnos reprobados esta representada con una línea roja, mostrando que su tendencia es la de disminuir. Con estos resultados fue posible realizar las ecuaciones correspondientes para poder demostrar de manera matemática si la tendencia de reprobación de los estudiantes asesorados incrementó o disminuyó. Por consiguiente, se presenta la Tabla no. 3 "asesorados aprobados" donde se han organizado los datos de tal manera que se pueda realizar el análisis matemático y así discernir si el número de estudiantes que fueron asesorados y obtuvieron aprobación aumentó o disminuyó.

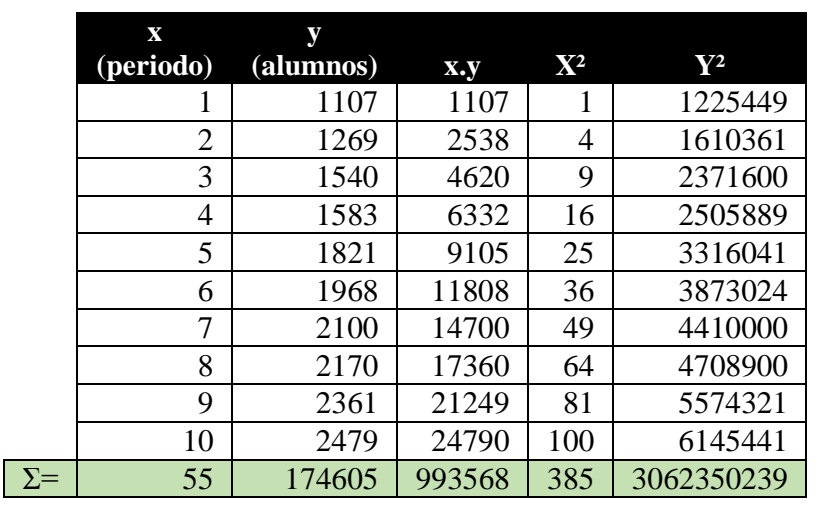

Tabla 3 "Asesorados aprobados"

Fuente: Elaboración Propia

Observando la tabla No. 3 y aplicando las fórmulas de la regresión lineal (Ecuación 1) se logró obtener la pendiente tendencia, la cual es el número que acompaña a la variable " $x$ ". Con esto se puede notar que dicho número es positivo, lo cual quiere decir que la tendencia de aprobación se incrementará conforme aumenten los periodos.

$Y=37.6364 x+1632.8$

\begin{tabular}{|c|c|c|c|c|}
\hline x (periodo) & y (alumnos) & $\mathbf{x y}$ & $\mathbf{X}^{2}$ & $\mathbf{Y}^{2}$ \\
\hline 1 & 240 & 240 & 1 & 57600 \\
\hline 2 & 171 & 342 & 4 & 29241 \\
\hline 3 & 244 & 732 & 9 & 59536 \\
\hline 4 & 194 & 776 & 16 & 37636 \\
\hline 5 & 195 & 975 & 25 & 38025 \\
\hline 6 & 163 & 978 & 36 & 26569 \\
\hline 7 & 169 & 1183 & 49 & 28561 \\
\hline 8 & 221 & 1768 & 64 & 48841 \\
\hline 9 & 194 & 1746 & 81 & 37636 \\
\hline 10 & 185 & 1850 & 100 & 34225 \\
\hline 55 & 1976 & 10590 & 385 & 397870 \\
\hline
\end{tabular}

Tabla 4 "Asesorados reprobados"

Fuente: Elaboración Propia

ISSN 2523-2509

ECORFAN® Todos los derechos reservados
De esta tabla y aplicando el mismo procedimiento se tiene que:

$Y=-0.8424 x+202.2333$

En la ecuación de la Tabla No 4., se puede observar que la variable equis tiene un prefijo negativo, esto nos indica que la pendiente es negativa además de que la tendencia si es disminuir.

\section{Conclusiones}

En los comportamientos de las gráficas y tablas, los estudios realizados nos han ayudado a comprender que entre las hipótesis de que "¿existe una relación entre brindar asesoramiento y la reducción del índice de reprobación dentro de la Dependencia? “¿es más probable que cuando un estudiante pida ayuda este pueda pasar?" se mostró la relación entre estudiantes asesorados y aprobados, lo cual daba como resultado menos estudiantes que reprobaban la materia.

Mientras el número de los asesorados iba creciendo, el número de los estudiantes aprobados también, por lo que el resultado obtenido durante este periodo 2014-2019, ha sido satisfactorio, incluso se podría plantear la idea de que con más estudiantes que se inscriban en el programa de Asesorías Académicas el índice de reprobación dentro de la FIME se podría aún reducir más.

Debido al impacto positivo que se ha logrado. El aumento de los alumnos aprobados y la disminución de los reprobados, indican que las asesorías académicas cumplen con el objetivo de ayudar, orientar y dar soporte a los estudiantes de la Dependencia

\section{Referencias}

Carreón Rodríguez, A., Díaz Camacho, S., Pérez Merlos, J., \& Salgado Gallegos, M. (2020). Disminución del índice de reprobación medianteestrategias tutoriales. Obtenido de https://revistas.uaa.mx/index.php/docere/article/ view/1778/1660

Corona, V., Reyes, S., Martínez, S., \& Rivas, C. (Diciembre de 2016). Revista de Sistemas y Gestión Educativa. Obtenido de http://www.ecorfan.org/bolivia/researchjournals /Sistemas_y_Gestion_Educativa/vol3num9/Sist emas_y_Gestion_Educativa_V3_N9.pdf

SORDIA-SALINAS, Cesar, PALOMARES-RUIZ, María Blanca, TORRES-BUGDUD, Arturo y TREVIÑO-CUBERO, Arnulfo. Análisis de la evolución de las Asesorías Académicas en el periodo 2014-2019 en una DES. Revista Teoría Educativa. 2020 
Fernández Fassnacht, E. (2017). Una mirada a los desafíos de la educación superior en México. Obtenido de http://www.scielo.org.mx/scielo.php?script=sci _arttext\&pid=S1665-

26732017000200183\&lng=es\&tlng=es

Franco, Y. G. (2013). Factores que intervienen en la reprobación de Matemáticas. España.

Gonzalo Nava Bustos, P. R. (2007). Factores de reprobacion en los alumnos del Centro Universitario de Ciencias de la Salud de la Universidad de Guadalajara. Revista de Educación y Desarrollo, 18.

Hoz, V. G. (1988). Educacion Personalizada. Madrid: Ediciones RIALP.

Roberto Hernández Sampieri, C. C. (2004). METODOLOGÍA DE LA INVESTIGACION. Mexico: McGraw-Hill Interamericana.

SEP. (2019). PRINCIPALES CIFRAS DEL SISTEMA EDUCATIVO NACIONAL. Obtenido de

https://www.planeacion.sep.gob.mx/Doc/estadi stica_e_indicadores/principales_cifras/principal es_cifras_2018_2019_bolsillo.pdf

Sordia C.S, Blanca, M., Palomares Ruiz, E., Rangel, D., \& Isabel, M. (2018). Multidisciplinas de la Ingeniería Fime Uanl Asesorías Académicas, Un Programa Institucional Que Fortalece El Desempeño De Una Des 176 Asesorías Académicas, Un Programa Institucional Que Fortalece El Desempeño De Una Des. https://www.multidisciplinasdelaingenieria.com /A\%C3\%B10\%20VI.\%20No\%207/PDF/Revist a7-176-182.pdf

Toscano de la Torre, B. A. (s.f.). La Eficiencia Terminal como un Indicador de la Calidad en la Educación Superior en México. Obtenido de La Universidad y sus Estrategias de Vinculacion: https://www.researchgate.net/publication/28884 1236_La_Eficiencia_Terminal_como_un_Indic ador_de_la_Calidad_en_la_Educacion_Superio r_en_Mexico

UNESCO. (12 de Octubre de 2020). UNESCO Future of Education - LEARNING TO BECOME. Obtenido de https://en.unesco.org/futuresofeducation/
Veronica Corona, S. R. (2016). Estrategias para la disminución de los índices de reprobación en el Instituto Tecnológico de Pachuca. Revista de Sistemas y Gestion Educativa, 62-69. 